\title{
LETTER
}

\section{A Primary Male A utosomal Linkage Map of the Horse Genome}

\section{Gabriella Lindgren, ${ }^{1,6}$ Kaj Sandberg, ${ }^{1}$ Helena Persson, ${ }^{1}$ Stefan Marklund, ${ }^{1,2}$ Matthew Breen, ${ }^{3}$ Björn Sandgren, ${ }^{4}$ Johan Carlstén, ${ }^{5}$ and Hans Ellegren ${ }^{1}$}

\author{
${ }^{1}$ Department of Animal Breeding and Genetics, Swedish University of Agricultural Sciences, S-751 24 \\ Uppsala, Sweden; ${ }^{2}$ Department of Animal Science, lowa State University, Ames, lowa 50011-3150 USA; \\ ${ }^{3}$ Centre for Preventive Medicine, Animal Health Trust, Newmarket, Suffolk CB8 7DW, UK; ${ }^{4}$ Bayer AB; \\ Veterinary Department, S-402 24 Göteborg, Sweden; ${ }^{5}$ Department of Clinical Radiology, Swedish \\ University of Agricultural Sciences, S-750 07 Uppsala, Sweden
}

\begin{abstract}
A primary male autosomal linkage map of the domestic horse (Equus caballus) has been developed by segregation analysis of $\mathbf{1 4 0}$ genetic markers within eight half-sib families. The family material comprised four Standardbred trotters and four Icelandic horses, with a total of 263 offspring. The marker set included $\mathrm{D} 1$ microsatellite markers, eight protein polymorphisms, five RFLPs, three blood group polymorphisms, two PCR-RFLPs, and one single strand conformation polymorphism (SSCP). O ne hundred markers were arranged into 25 linkage groups, 22 of which could be assigned physically to 18 different chromosomes (ECA 1, ECA 2, ECA 3, ECA 4, ECA 5, ECA 6, ECA 7, ECA 9, ECA 10, ECA 11, ECA 13, ECA 15, ECA 16, ECA 18, ECA 19, ECA 21, ECA 22, and ECA 30). The average distance between linked markers was $D .6 \mathrm{cM}$ and the longest linkage group measured $103 \mathrm{cM}$. The total map distance contained within linkage groups was $679 \mathrm{cM}$. If the distances covered outside the ends of linkage groups and by unlinked markers were included, it was estimated that the marker set covered at least $1500 \mathrm{cM}$, that is, at least $50 \%$ of the genome. A comparison of the relationship between genetic and physical distances in anchored linkage groups gave ratios of $0.5-0.8 \mathrm{cM}$ per $\mathrm{Mb}$ of DNA. This would suggest that the total male recombinational distance in the horse is $2000 \mathrm{cM}$; this value is lower than that suggested by chiasma counts. The present map should provide an important framework for future genome mapping in the horse.
\end{abstract}

Following a general trend in modern genetics, domestic animal genome analysis has witnessed some considerable achievements over the last 5-10 years, achievements that not least can be attributed to the advent and introduction of new DNA marker technology. Significantly, reports are accumulating on the identification of genes or chromosomal regions that influence quantitative or qualitative traits of economic, agricultural, or even medical importance. Prime examples include malignant hyperthermia (MacLennan et al. 1990) and fatness (Andersson et al. 1994) in pigs, milk production (Georges et al. 1995) and double muscling (Charlier et al. 1995) in cattle, and fecundity in sheep (Montgomery et al. 1994). Identification of such loci has been mediated by the initial construction of genome maps based on genetic linkage data or physical assignments of markers to chromosomes. Pri-

${ }^{6}$ Corresponding author.

E-MAIL Gabriella.Lindgren@bmc.uu.se; FAX 46-18-504461. mary or in some cases even second-generation linkage maps are now available for most domestic animal species, for example, cattle (Barendse et al. 1997; Kappes et al. 1997), pig (Marklund et al. 1996a; Rohrer et al. 1996), sheep (Crawford et al. 1995), goat (Vaiman et al. 1996), and dog (Mellersh et al. 1997).

In terms of genetic map information as well as of other aspects of genome analysis, one main domestic animal species has been clearly lagging behind the others-the horse. There is no primary linkage map reported for the horse. The last compilation of incidental cases of observed linkages, mainly involving protein polymorphisms, listed only 17-20 loci, few of which had been assigned to any of the 31 equine autosomes (Sandberg and Andersson 1992). The first equine microsatellite markers were derived some five years ago (Ellegren et al. 1992), but a large set of markers has not become available until recently. A few linkage groups derived from the preliminary analyses of limited 
numbers of microsatellites are known (e.g., Marklund et al. 1994), and initial efforts to integrate genetic and physical map data have been made (Breen et al. 1997; Godard et al. 1997). The reasons for the slow progress in equine gene mapping are several and relate mainly to the economy and practice of horse breeding (but potentially also to the fact that the horse in some cases is seen more as a companion species than as livestock). Horse breeding is associated with extensive costs and demands (personnel, stables, grazing areas), and since a large number (>100-200) of animals might be required for the construction of linkage maps, suitable pedigree might be difficult to establish. Moreover, as the horse has a gestation period of 11 months and generally produces a single foal per mating, designing large full-sib families is problematic. Thus, in practice, equine linkage mapping has to rely on family material available for other reasons than genetic studies, one typical example being large (twogeneration) half-sib families present for popular stallions. Generally, such materials represent within-breed matings, that is, matings not associated with an optimized marker heterozygosity.

In an initiative to extend the knowledge of the equine genome, we have established a reference pedigree for gene mapping and we present here the construction of the first preliminary, male autosomal linkage map of the horse. The map was derived from the genotyping of 140 polymorphic markers, 100 of which were arranged into 25 linkage groups on 18 different autosomes.

\section{RESULTS}

Genetic Markers

We designed an equine reference pedigree for the purpose of gene mapping, involving eight half-sib families (four Standardbred and four Icelandic Horse stallions) and a total of 263 offspring. The pedigree was genotyped with 140 polymorphic markers, 121 of which were PCR-based microsatellites and about onethird of which had been assigned physically to chromosomes by in situ hybridization (Table 1). The markers included five new, previously undescribed restriction fragment length polymorphisms (RFLPs) that were detected using human or porcine CDNA probes, FUCA1, GLUT1, $L P L, M Y L 1,3$, and TYR; data for these polymorphisms are presented in Table 2.

Marker heterozygosity varied extensively between loci, from one out of the eight sires being heterozygote to all being so. The average number of heterozygous sires was 4.3, which corresponds to an observed heterozygosity $\left(H_{0}\right)$ of $53 \%$. In general, microsatellites were more variable than the other markers in this study ( $\mathrm{H}_{\mathrm{o}}=56 \%$ vs. $\left.35 \%\right)$. Two important consequences of only the sires and their offspring being included in the study were that we only measured male recombination fractions and could not deduce the transmission of paternal alleles for all offspring. In a heterozygous offspring showing the same two alleles as its father, knowledge of the genotype of its mother is required for following the inheritance of the sire's allele. The average number of fully informative offspring per marker was 89, ranging from 9 to 235 .

\section{Linkage A nalysis}

An overview of the linkage data is given in Figure 1, where the equine idiogram (Bowling et al. 1997a) is shown together with the established linkage groups. One hundred of the 140 markers (72\%) showed linkage to at least one other marker. The average pair-wise recombination fraction between linked markers, using an lod score of three as threshold level for significant linkage, was $9.7 \% \pm 0.7$ S.E., with $26 \%$ as the longest interval for which linkage was detected (ASB6-ASB9). By multipoint analysis we could establish 25 linkage groups. Twenty-two of these were assigned to 18 different chromosomes, generally caused by one or more of the markers included in a linkage group being mapped physically by in situ hybridization (see legend to Fig. 1). Chromosomes covered by linkage groups were ECA1, ECA2, ECA3, ECA4, ECA5, ECA6, ECA7, ECA9, ECA10, ECA11, ECA13, ECA15, ECA16, ECA18, ECA19, ECA21, ECA22, and ECA30. For four additional chromosomes (ECA12, ECA20, ECA23, and ECA26), a physically anchored genetic marker was included in our material but showed no linkage to other markers. Only two metacentric (ECA8 and ECA12) and two of the larger acrocentric chromosomes (ECA14 and ECA17) were not tagged by physically assigned linkage groups.

Linkage groups ranged between 0 and $103 \mathrm{cM}$ (multipoint distances in Kosambi cM), the longest residing on ECA4, and the number of markers within groups between 2 and 10 . The order of markers within linkage groups could in several cases not be resolved with confidence (i.e., the odds against reversed order of adjacent markers being lower than 1000:1), and the precise order of markers in such groups should therefore be regarded as tentative. For the purpose of this study, however, we consider it important to report even tentative orders in light 
Table 1. Description of Markers Used in this Study

\begin{tabular}{|c|c|c|c|c|c|}
\hline Locus & Description & $\begin{array}{c}\text { Primer } \\
\text { sequences }\end{array}$ & $\mathbf{H}_{\mathbf{0}}{ }^{\mathrm{a}}$ & $\begin{array}{l}\text { Phys. } \\
\text { assign. }\end{array}$ & Reference \\
\hline \multicolumn{6}{|c|}{$\begin{array}{l}\text { Genes } \\
\text { Blood groups }\end{array}$} \\
\hline EAA & erythrocyte antigen A & & 50 & & Bailey et al. (1979) \\
\hline EAD & erythrocyte antigen $\mathrm{D}$ & & 37.5 & & Sandberg (1973) \\
\hline EAQ & erythrocyte antigen Q & & 37.5 & & Bowling et al. (1985) \\
\hline \multicolumn{6}{|c|}{ Microsatellite } \\
\hline IGF2 & $\begin{array}{l}\text { insulin-like } \\
\text { growth factor } 2\end{array}$ & $\begin{array}{l}\text { GGAGCACAGAACATGAAAAC } \\
\text { AAATTTAATTGGCACAAACC }\end{array}$ & 87.5 & $12 q 13$ & $\begin{array}{l}\text { H. Elegren (unpubl.) } \\
\text { Raudsepp et al. } \\
\text { (1997) }\end{array}$ \\
\hline \multicolumn{6}{|c|}{ PCR-RFLPs } \\
\hline $\mathrm{MClR}$ & $\begin{array}{l}\text { melanocyte- } \\
\text { stimulating } \\
\text { hormone } \\
\text { receptor } 1\end{array}$ & $\begin{array}{l}\text { GATGGATCCTTCTGGGCTCCCTCAACTC } \\
\text { GTAGTAGGCGATGAAGAGCGTGCT }\end{array}$ & 37.5 & $3 p 12$ & $\begin{array}{l}\text { Marklund et al. } \\
\text { (1996b) } \\
\text { T. Raudsepp } \\
\text { (unpubl.) }\end{array}$ \\
\hline KIT & $\begin{array}{l}\text { mast cell growth } \\
\text { factor receptor }\end{array}$ & $\begin{array}{l}\text { ATTTATTCCAACTTAGCGAACTGCAGC } \\
\text { TCAGACATCTTCGTGGACAAGCAGAGG }\end{array}$ & 25 & $3 q 21$ & $\begin{array}{l}\text { Marklund et al. } \\
\text { (1996b) } \\
\text { Lear et al. (1997) }\end{array}$ \\
\hline \multicolumn{6}{|l|}{ Proteins } \\
\hline AlBG & $\alpha$-1-B glycoprotein & & 37.5 & & Juneja et al. (1987) \\
\hline ALB & albumin & & 50 & $3 q 14.3$ & $\begin{array}{l}\text { Andersson and } \\
\text { Sandberg (1982) } \\
\text { Godard et al. (1998) }\end{array}$ \\
\hline $\mathrm{CA}$ & carbonic anhydrase & & 37.5 & & $\begin{array}{l}\text { Sandberg et al. } \\
\text { (1968) }\end{array}$ \\
\hline ES & carboxylesterase & & 50 & & $\begin{array}{l}\text { Andersson and } \\
\text { Sandberg (1982) }\end{array}$ \\
\hline HBA & hemoglobin A & & 37.5 & 13pter & $\begin{array}{l}\text { Bowling et al. (1988) } \\
\text { Oakenfull et al. } \\
\text { (1993) }\end{array}$ \\
\hline PGD & $\begin{array}{c}\text { 6-phosphogluconate } \\
\text { dehydrogenase }\end{array}$ & & 25 & $2 p 12-13$ & $\begin{array}{l}\text { Andersson et al. } \\
\text { (1984) } \\
\text { Gu et al. (1992) }\end{array}$ \\
\hline PGM & phosphoglucomutase & & 37.5 & & $\begin{array}{l}\text { Dawson and Jaeger } \\
\text { (1970) }\end{array}$ \\
\hline $\begin{array}{l}\text { TF } \\
\text { RFPs }\end{array}$ & transferrin & & 50 & & Gahne (1966) \\
\hline FUCA & $\alpha-L-1$ fucosidase & & 37.5 & & this study \\
\hline GLUT & $\begin{array}{l}\text { glucose transporter } \\
\text { type } 1\end{array}$ & & 12.5 & & this study \\
\hline LPL & lipoprotein lipase & & 12.5 & & this study \\
\hline MYL & $\begin{array}{l}\text { myosin light } \\
\text { polypeptide 1, } 3\end{array}$ & & 75 & & this study \\
\hline ELA-DRB & $\begin{array}{l}\text { equine leucocyte } \\
\text { antigen class II }\end{array}$ & $\begin{array}{l}\text { CTCTGCAGCACATTTCCTGGAG } \\
\text { CGCCGCTGCACCAGGAA }\end{array}$ & 25 & $20 q 14$ & $\begin{array}{l}\text { Fraser and Bailey } \\
\quad(1996) \\
\text { Ansari et al. (1988) }\end{array}$ \\
\hline \multicolumn{6}{|c|}{ Anonymous loci } \\
\hline A-14 & microsatellite & $\begin{array}{l}\text { CAGCTGGGTGACACAGAGAG } \\
\text { GTCATCACTACTCCCTACAC }\end{array}$ & 75 & $2 q 14-21$ & Marti et al. (1998) \\
\hline A-17 & microsatellite & $\begin{array}{l}\text { GTGGAGAGATAAAAGAAGATCC } \\
\text { GGCCACAAGGAATGAACACAC }\end{array}$ & 50 & $26 q 13-14$ & Marti et al. (1998) \\
\hline ASB1 & microsatellite & $\begin{array}{l}\text { AGCAGAAACCCACTCAAGCC } \\
\text { GCATAATACCCTCAAGGTC }\end{array}$ & 75 & & Breen et al. (1997) \\
\hline ASB2 & microsatellite & $\begin{array}{l}\text { CCTTCCGTAGTITAAGCTTCTG } \\
\text { CACAACTGAGTTCTCTGATAGG }\end{array}$ & 87.5 & $15 q 21.3-23$ & Breen et al. (1997) \\
\hline
\end{tabular}


Table 1. (Continued)

\begin{tabular}{|c|c|c|c|c|c|}
\hline Locus & Description & $\begin{array}{c}\text { Primer } \\
\text { sequences }\end{array}$ & $\mathrm{H}_{\mathbf{0}}{ }^{\mathrm{a}}$ & $\begin{array}{l}\text { Phys. } \\
\text { assign. }\end{array}$ & Reference \\
\hline ASB3 & microsatellite & $\begin{array}{l}\text { AATTCATCTCAGTGCTCTACCAGC } \\
\text { TTCATIITCTACATGCACTACAGC }\end{array}$ & 87.5 & $4 q 12-13$ & Breen et al. (1997) \\
\hline ASB4 & microsatellite & $\begin{array}{l}\text { TAAATTGTAAAAGCTGGAGCCG } \\
\text { GCAAATAGTAGTTAAGTCCTC }\end{array}$ & 100 & $9 q 16-18$ & Breen et al. (1997) \\
\hline ASB5 & microsatellite & $\begin{array}{l}\text { TCGAGGAGCTCATGACCTGG } \\
\text { TTGTACAACTCTCCACCATAGC }\end{array}$ & 75 & $9 q 16-18$ & Breen et al. (1997) \\
\hline ASB6 & microsatellite & $\begin{array}{l}\text { GGCACAGATGTTAGCTCAGC } \\
\text { ATGGAACCAGCCTGGATTGC }\end{array}$ & 75 & 10p13 & Breen et al. (1997) \\
\hline ASB7 & microsatellite & $\begin{array}{l}\text { CTGGAAATTACAGTGGTCTTCTGG } \\
\text { AGGTIITCAGGGGCTTGCGAAGC }\end{array}$ & 62.5 & $19 q 14-16$ & Breen et al. $(1997)^{\mathrm{b}}$ \\
\hline ASB8 & microsatellite & $\begin{array}{l}\text { GACAACGTGGCAGCTCACTGCC } \\
\text { GCAAGTAAGCCATATGTGCATGCG }\end{array}$ & 37.5 & $1 q 16-17$ & Breen et al. (1997) \\
\hline ASB9 & microsatellite & $\begin{array}{l}\text { GTGCGCATGTATGTGCGTGCC } \\
\text { ATTCCACAAGGGACATGAGG }\end{array}$ & 75 & $10 q 21-23$ & Breen et al. (1997) \\
\hline ASB10 & microsatellite & $\begin{array}{l}\text { GTTGTCTAGGTGCAGAATCTGG } \\
\text { GTTATGTCTCCCCTTCTCTACC }\end{array}$ & 75 & & Breen et al. (1997) \\
\hline ASB11 & microsatellite & $\begin{array}{l}\text { CCACCTATGTGTTCAGTTCACC } \\
\text { GCACCAATGTTATAGACTCCC }\end{array}$ & 50 & $19 q 21-22$ & Breen et al. $(1997)^{\mathrm{b}}$ \\
\hline ASB12 & microsatellite & $\begin{array}{l}\text { TCAGCAATAGAAGCCAGCTCC } \\
\text { TCCTATGGAGGTGACCTTCCC }\end{array}$ & 75 & $1 q 12-13$ & Breen et al. (1997) \\
\hline ASB13 & microsatellite & $\begin{array}{l}\text { CTCTGAAAGAGCAGGATTGG } \\
\text { GTCTTCTAAGTGGTAAGAGCC }\end{array}$ & 37.5 & $2 q 14.3-21.2$ & Breen et al. $(1997)^{\mathrm{b}}$ \\
\hline ASB14 & microsatellite & $\begin{array}{l}\text { CTCCATGAATTCTCGCAGGTTGG } \\
\text { CCATGGGCCATATGCACACTGC }\end{array}$ & 25 & $6 q 21$ & Breen et al. (1997) \\
\hline ASB15 & microsatellite & $\begin{array}{l}\text { GTCCCAAAGGGACTCAGGAAGG } \\
\text { TGGATGCCAGTGCATAGACAG }\end{array}$ & 37.5 & $15 q 21$ & Breen et al. (1997) \\
\hline ASB17 & microsatellite & $\begin{array}{l}\text { GAGGGCGGTACCTTTGTACC } \\
\text { ACCAGTCAGGATCTCCACCG }\end{array}$ & 62.5 & 2p14-15 & Breen et al. (1997) \\
\hline ASB18 & microsatellite & $\begin{array}{l}\text { TGCAGACAAAGCTGGACACTC } \\
\text { CTGCTGAGAAAGCTTCTGC }\end{array}$ & 50 & & Breen et al. (1997) \\
\hline ASB19 & microsatellite & $\begin{array}{l}\text { GAGTTGGAGCTCAAGTCTGTC } \\
\text { GTITAGCAACTACAGCGTAGG }\end{array}$ & 62.5 & $15 q 21.3-23$ & Breen et al. (1997) \\
\hline ASB22 & microsatellite & $\begin{array}{l}\text { GAGGAATGTGAAATACAGGAGG } \\
\text { TITGTGGTCTTCCGTGCACC }\end{array}$ & 75 & $4 q 21$ & Breen et al. (1997) \\
\hline ASB23 & microsatellite & $\begin{array}{l}\text { GAGGTTTGTAATTGGAATG } \\
\text { GAGAAGTCATITTAACACCT }\end{array}$ & 62.5 & $3 q 22$ & $\begin{array}{l}\text { Irvin et al. (1998) } \\
\text { Lear et al. (1998) }\end{array}$ \\
\hline ASB35 & microsatellite & $\begin{array}{l}\text { ATGCATGAGCAGAGTGTTCTTCC } \\
\text { TAGTACTTCTCTCTTAATATAAGC }\end{array}$ & 62.5 & & $\begin{array}{l}\text { M. Breen et al. } \\
\text { (unpubl.) }\end{array}$ \\
\hline ASB36 & microsatellite & $\begin{array}{l}\text { GAACATGTAGTGTTTACTCTGCC } \\
\text { GAAGGTTGTGGGTCTTACAAGG }\end{array}$ & 50 & & $\begin{array}{l}\text { M. Breen et al. } \\
\text { (unpubl.) }\end{array}$ \\
\hline ASB37 & microsatellite & $\begin{array}{l}\text { CCTGCAACTTITCCCAGCC } \\
\text { GGCAGATGTTAGCTCATGGC }\end{array}$ & 12.5 & $13 q 11-12$ & $\begin{array}{l}\text { Invin et al. (1998) } \\
\text { Lear et al. (1998) }\end{array}$ \\
\hline ASB38 & microsatellite & $\begin{array}{l}\text { TGGGGTTGCCTTGGTTACC } \\
\text { TCAGAGGATGAGGCACAGC }\end{array}$ & 62.5 & & Irvin et al. (1998) \\
\hline B-8 & microsatellite & $\begin{array}{l}\text { TCCTCAGTCCTTTCTCATGC } \\
\text { AGCTGAAGGCAATCTGTACC }\end{array}$ & 62.5 & $15 q 14-21$ & Marti et al. (1998) \\
\hline D-8 & microsatellite & $\begin{array}{l}\text { TIITGTGTCTCAGGAGTGTG } \\
\text { AGTCTGATGGTGGAGGAAGG }\end{array}$ & 62.5 & $11 p 12-13$ & Marti et al. (1998) \\
\hline ECA-2 & microsatellite & $\begin{array}{l}\text { TTCCCTCCCATGGTTATTITTC } \\
\text { TCTCTACTITCATATACATTGG }\end{array}$ & 100 & $(1 \mathrm{q} 2.1)^{\mathrm{c}}$ & $\begin{array}{l}\text { Sakagami et al. } \\
\text { (1995) }\end{array}$ \\
\hline ECA-3 & microsatellite & $\begin{array}{l}\text { GGTTCACACAGGAGTCAGGGA } \\
\text { CCTTCTGGTTGCCTCGTCTC }\end{array}$ & 50 & $(2 \mathrm{p} 1.3-4)^{c}$ & Tozaki et al. (1995) \\
\hline HLM 3 & microsatellite & $\begin{array}{l}\text { GAAGGTAGAAAAGGAGGGCTAGAAC } \\
\text { TCTAGAGGACCATTCTCTGGGCTGTG }\end{array}$ & 12.5 & & Vega-Pla et al. (1996) \\
\hline HMB1 & microsatellite & $\begin{array}{l}\text { GTGTGTATGCTTCCCAACCCTT } \\
\text { GTTATAAAGCACTATGATCTCA }\end{array}$ & 50 & & Binns et al. (1995) \\
\hline
\end{tabular}




\begin{tabular}{|c|c|c|c|c|c|}
\hline Locus & Description & $\begin{array}{l}\text { Primer } \\
\text { sequences }\end{array}$ & $\mathrm{Ho}^{\mathrm{a}}$ & $\begin{array}{l}\text { Phys. } \\
\text { assign. }\end{array}$ & Reference \\
\hline HM B2 & microsatellite & $\begin{array}{l}\text { GTGCCACCACCTCTGTGATT } \\
\text { TGGAGAAGGATCTTGGCTC }\end{array}$ & 50 & & Binns et al. (1995) \\
\hline HMB3 & microsatellite & $\begin{array}{l}\text { CAAACATCAGTTAAGAGTGA } \\
\text { CTCTAATCCAGCAGTGTTCA }\end{array}$ & 75 & & Binns et al. (1995) \\
\hline HMB4 & microsatellite & $\begin{array}{l}\text { AACCGCCTGAGCAAGGAAGT } \\
\text { GCTCCCAGAGAGTITACCCT }\end{array}$ & 62.5 & & Binns et al. (1995) \\
\hline HM B5 & microsatellite & $\begin{array}{l}\text { ACGGACACATCCCTGCCTGC } \\
\text { GCAGGCTAAGGAGGCTCAGC }\end{array}$ & 75 & & Binns et al. (1995) \\
\hline HM B6 & microsatellite & $\begin{array}{l}\text { GAAGATGTCCGCTTTGATAT } \\
\text { CACTGGCACATCCAGATTG }\end{array}$ & 75 & & Binns et al. (1995) \\
\hline HMS1 & microsatellite & $\begin{array}{l}\text { CATCACTCTTCATGTCTGCTTGG } \\
\text { TTGACATAAATGCTTATCCTATGGC }\end{array}$ & 62.5 & & Guérin et al. (1994) \\
\hline HMS2 & microsatellite & $\begin{array}{l}\text { ACGGTGGCAACTGCCAAGGAAG } \\
\text { CTTGCAGTCGAATGTGTATTAAATG }\end{array}$ & 62.5 & & Guérin et al. (1994) \\
\hline HMS3 & microsatellite & $\begin{array}{l}\text { CCAACTCTTTGTCACATAACAAGA } \\
\text { CAATCCTCACTIITCACTITGT }\end{array}$ & 62.5 & & Guérin et al. (1994) \\
\hline HM S5 & microsatellite & $\begin{array}{l}\text { TAGTGTATCCGTCAGAGTTCAAAG } \\
\text { GCAAGGAAGTCAGACTCCTGGA }\end{array}$ & 62.5 & & Guérin et al. (1994) \\
\hline HM S6 & microsatellite & $\begin{array}{l}\text { GAAGCTGCCAGTATTCAACCATTG } \\
\text { CTCCATCTTGTGAAGTGTAACTCA }\end{array}$ & 75 & & Guérin et al. (1994) \\
\hline HMS7 & microsatellite & $\begin{array}{l}\text { CAGGAAACTCATGTTGATACCATC } \\
\text { TGTTGTTGAAACATACCTTGACTGT }\end{array}$ & 75 & & Guérin et al. (1994) \\
\hline HMS18 & microsatellite & $\begin{array}{l}\text { CAACAATGAAAATTGTCCTGTGC } \\
\text { GTAAATGAGTAGACAATCATGAGG }\end{array}$ & 62.5 & & Godard et al. (1997) \\
\hline HMS19 & microsatellite & $\begin{array}{l}\text { CTAACCAGCACAGAATGAATGGC } \\
\text { TAAAAGAACAGTGGAGAGTAAAGTG }\end{array}$ & 25 & $4 q 21$ & $\begin{array}{l}\text { Godard et al. (1997, } \\
\text { 1998) }\end{array}$ \\
\hline HMS20 & microsatellite & $\begin{array}{l}\text { TGGGAGAGGTACCTGAAATGTAC } \\
\text { GTTGCTATAAAAAATTTCTCCCTAC }\end{array}$ & 100 & & $\begin{array}{l}\text { Guérin and Bertaud } \\
\text { (1996) }\end{array}$ \\
\hline HMS23 & microsatellite & $\begin{array}{l}\text { GATCCAATATTGTAAACCCCGCC } \\
\text { CCTTCATAACCCTTATTGCAGCC }\end{array}$ & 37.5 & & Godard et al. (1997) \\
\hline HMS45 & microsatellite & $\begin{array}{l}\text { TGTTACAGGTATTGGTAAACTGTGC } \\
\text { GGAACAAGAAGAAATCACTAATGTC }\end{array}$ & 75 & & Godard et al. (1997) \\
\hline HMS46 & microsatellite & $\begin{array}{l}\text { GTCTCAGCCAAAAGGTATTCAAGC } \\
\text { TGGCACCAATATAGGTCACCTGG }\end{array}$ & 50 & & Godard et al. (1997) \\
\hline HMS47 & microsatellite & $\begin{array}{l}\text { CCTGCTGAGGACCTTGGAAGCT } \\
\text { ATGTATITCAAGTCTAATATCTGCC }\end{array}$ & 25 & $22 q 19$ & $\begin{array}{l}\text { Godard et al. (1997, } \\
\text { 1998) }\end{array}$ \\
\hline HTG2 & microsatellite & $\begin{array}{l}\text { GATTGGCAACAGATGTTAACTCGG } \\
\text { CCCCATGAGAACTAACAATGTTAG }\end{array}$ & 12.5 & & Ellegren et al. (1992) \\
\hline HTG3 & microsatellite & $\begin{array}{l}\text { TAACCTGGGTGCAAAGCCACCCAT } \\
\text { TCAGGGCCAATCTCCTCAC }\end{array}$ & 62.5 & & Ellegren et al. (1992) \\
\hline HTG4 & microsatellite & $\begin{array}{l}\text { CTATCTCAGTCTTCATTGCAGGAC } \\
\text { CTCCCTCCCTCCCTCTGTTCTC }\end{array}$ & 100 & & Ellegren et al. (1992) \\
\hline HTG5 & microsatellite & $\begin{array}{l}\text { TGCTAAGCCTCAGCACATACA } \\
\text { TGGAAATAAGGTAGCAGGGATGC }\end{array}$ & 50 & & Ellegren et al. (1992) \\
\hline HTG6 & microsatellite & $\begin{array}{l}\text { CCTGCTTGGAGGCTGTGATAAGAT } \\
\text { GTTCACTGAATGTCAAATTCTGCT }\end{array}$ & 37.5 & $15 q 26-27$ & $\begin{array}{l}\text { Ellegren et al. (1992) } \\
\text { Godard et al. (1998) }\end{array}$ \\
\hline HTG7 & microsatellite & $\begin{array}{l}\text { CCTGAAGCAGAACATCCCTCCTTG } \\
\text { ATAAAGTGTCTGGGCAGAGCTGCT }\end{array}$ & 25 & & $\begin{array}{l}\text { Marklund et al. } \\
\text { (1994) }\end{array}$ \\
\hline HTG8 & microsatellite & $\begin{array}{l}\text { CAGGCCGTAGATGACTACCAATGA } \\
\text { IITCAGAGTTAATTGGTATCACA }\end{array}$ & 62.5 & & $\begin{array}{l}\text { Marklund et al. } \\
\text { (1994) }\end{array}$ \\
\hline HTG9 & microsatellite & $\begin{array}{l}\text { TGTGGGAAGAGTGTCAATAGCTGT } \\
\text { AGGCATCTGGTTGCTGCAATTTC }\end{array}$ & 62.5 & $4 q 21.3$ & $\begin{array}{l}\text { Marklund et al. } \\
\text { (1994) } \\
\text { Godard et al. (1998) }\end{array}$ \\
\hline HTG10 & microsatellite & $\begin{array}{l}\text { CAATTCCCGCCCCACCCCCGGCA } \\
\text { TIITATTCTGATCTGTCACATTT }\end{array}$ & 87.5 & & $\begin{array}{l}\text { Marklund et al. } \\
\text { (1994) }\end{array}$ \\
\hline
\end{tabular}


LINDGREN ET AL.

Table 1. (Continued)

\begin{tabular}{|c|c|c|c|c|c|}
\hline Locus & Description & $\begin{array}{c}\text { Primer } \\
\text { sequences }\end{array}$ & $\mathbf{H}_{0}{ }^{a}$ & $\begin{array}{l}\text { Phys. } \\
\text { assign. }\end{array}$ & Reference \\
\hline HTG11 & microsatellite & $\begin{array}{l}\text { CAATGATGGTACTTTGCATATTAA } \\
\text { ATCGGCATGCACACTCATAGGTAG }\end{array}$ & 37.5 & & $\begin{array}{l}\text { Marklund et al. } \\
\text { (1994) }\end{array}$ \\
\hline HTG12 & microsatellite & $\begin{array}{l}\text { CACTAGAGTCAGGGGGGGTGGGCT } \\
\text { TTGGAGTACTCTITCTCCCTTCCC }\end{array}$ & 12.5 & & $\begin{array}{l}\text { Marklund et al. } \\
\text { (1994) }\end{array}$ \\
\hline HTG13 & microsatellite & $\begin{array}{l}\text { TTAGCACGGGGAGATCGGATCCTG } \\
\text { GGTCTCCCTCTCCATTCACCCTGC }\end{array}$ & 75 & & $\begin{array}{l}\text { Marklund et al. } \\
\text { (1994) }\end{array}$ \\
\hline HTG14 & microsatellite & $\begin{array}{l}\text { CCAGTCTAAGTTTGTTGGCTAGAA } \\
\text { CAAAGGTGAGTGATGGATGGAAGC }\end{array}$ & 62.5 & & $\begin{array}{l}\text { Marklund et al. } \\
\text { (1994) }\end{array}$ \\
\hline HTG15 & microsatellite & $\begin{array}{l}\text { TCTTGATGGCAGAGCCAGGATTTG } \\
\text { AATGTCACCATGCGGCACATGACT }\end{array}$ & 37.5 & & $\begin{array}{l}\text { Marklund et al. } \\
\text { (1994) }\end{array}$ \\
\hline HTG17 & microsatellite & $\begin{array}{l}\text { GCTATCCCTCCTGAGTCTTA } \\
\text { AGGTAATTTGAAATAAAATACAC }\end{array}$ & 87.5 & & $\begin{array}{l}\text { Lindgren et al. } \\
\text { (1998) }\end{array}$ \\
\hline $1-12$ & microsatellite & $\begin{array}{l}\text { AACTAAGCACGTCATACAAG } \\
\text { CTTGTAGTIITCGTTGTATAGC }\end{array}$ & 37.5 & $19 q 12-14$ & Marti et al. (1998) \\
\hline I-18 & microsatellite & $\begin{array}{l}\text { CAACAAAGATGTTGCAAGGG } \\
\text { TGTGCCTCTTGTCTCTTAGG }\end{array}$ & 50 & $16 q 23-25$ & Marti et al. (1998) \\
\hline LEX2 & microsatellite & $\begin{array}{l}\text { AAAAGGAAGACTGGCGACAG } \\
\text { GGTGGGGGAAAGAATGGT }\end{array}$ & 12.5 & & Coogle et al. (1996a) \\
\hline LEX4 & microsatellite & $\begin{array}{l}\text { AATAGCAAATCTCCCACTTCA } \\
\text { GTCCTCACAACCTCATCATAA }\end{array}$ & 25 & & Coogle et al. (1996a) \\
\hline LEX5 & microsatellite & $\begin{array}{l}\text { AAGGCAATGCTTATCAAATGC } \\
\text { TTACCCGCAGTGACTTCTATT }\end{array}$ & 50 & & Coogle et al. (1996a) \\
\hline LEX7 & microsatellite & $\begin{array}{l}\text { GGTAGGGCTCTGGGATGA } \\
\text { AACACTGGGGAAAAGTCAG }\end{array}$ & 50 & & Coogle et al. (1996a) \\
\hline LEX8 & microsatellite & $\begin{array}{l}\text { AAACTGTCACAACGGTTAGGAC } \\
\text { CGAAAAAGCCACTTGAGGTC }\end{array}$ & 37.5 & & Coogle et al. (1996a) \\
\hline LEX9 & microsatellite & $\begin{array}{l}\text { AAAGCCGTAAGATTGGGACA } \\
\text { TCCATTGTGAGGGTGTAACA }\end{array}$ & 75 & & Coogle et al. (1996a) \\
\hline LEX11 & microsatellite & $\begin{array}{l}\text { ATTCCCAGTGAAGTATTGCCA } \\
\text { AGAGATGGGTACCTGGGATTC }\end{array}$ & 37.5 & & Coogle et al. (1996a) \\
\hline LEX14 & microsatellite & $\begin{array}{l}\text { CCTTACTCACTGGGGAATAAA } \\
\text { AGACTGAACACCTAACTATGA }\end{array}$ & 87.5 & & Coogle et al. (1996a) \\
\hline LEX15 & microsatellite & $\begin{array}{l}\text { GCATTCCCATCATCACAT } \\
\text { CCTGCCTTGCCTCTTCT }\end{array}$ & 37.5 & & Coogle et al. (1996b) \\
\hline LEX16 & microsatellite & $\begin{array}{l}\text { GTGGGGCCGGTATAGTGATTG } \\
\text { ACCCTAACTGATAACTGATAGA }\end{array}$ & 50 & & Coogle et al. (1996b) \\
\hline LEX17 & microsatellite & $\begin{array}{l}\text { CCTGCCCAAGAAGAACTCAGA } \\
\text { AGCAGTGTATITITGAAACAT }\end{array}$ & 100 & & Coogle et al. (1996b) \\
\hline LEX18 & microsatellite & $\begin{array}{l}\text { TTCATCACTTTCTGCTTCC } \\
\text { TTCTCTCCTITGCTCATCCT }\end{array}$ & 25 & & Coogle et al. (1996b) \\
\hline LEX19 & microsatellite & $\begin{array}{l}\text { TTCCCTITCCTCACATCCT } \\
\text { TITAGGTTCATCTATGTTGTTGC }\end{array}$ & 87.5 & & Coogle et al. (1996b) \\
\hline LEX20 & microsatellite & $\begin{array}{l}\text { GGAATAGGTGGGGGTCTGTT } \\
\text { AGGGTACTAGCCAAGTGACTGC }\end{array}$ & 37.5 & & Coogle et al. (1996b) \\
\hline LEX21 & microsatellite & $\begin{array}{l}\text { GTAGGCTTICTGCCAAAAT } \\
\text { TGAGGGGAGTCATAAAAA }\end{array}$ & 62.5 & & Coogle et al. (1996b) \\
\hline LEX22 & microsatellite & $\begin{array}{l}\text { AACATATCCATCGCCTCACA } \\
\text { TGCAAATTCACTGAGAGTGG }\end{array}$ & 25 & & Coogle et al. (1996b) \\
\hline LEX23 & microsatellite & $\begin{array}{l}\text { GGATGAAACAGGGAAGGAAA } \\
\text { CCAACGGATTCATGAAAGCTA }\end{array}$ & 62.5 & & Coogle et al. (1996b) \\
\hline LEX25 & microsatellite & $\begin{array}{l}\text { CAATCGTGGCCCGGTAAC } \\
\text { TTCACTCCAATCCTCAGTCA }\end{array}$ & 50 & & Coogle et al. (1996c) \\
\hline LEX27 & microsatellite & $\begin{array}{l}\text { ACCACTGGGAAACTGTGTAA } \\
\text { GCCCAGAATCCGAACC }\end{array}$ & 25 & & Coogle et al. (1996c) \\
\hline LEX29 & microsatellite & $\begin{array}{l}\text { TGGGGTGTCACTGCTTCTC } \\
\text { ACTGAGGGCCAGGTTTCTAA }\end{array}$ & 62.5 & & Coogle et al. (1996c) \\
\hline
\end{tabular}


Table 1. (Continued)

\begin{tabular}{|c|c|c|c|c|c|}
\hline Locus & Description & $\begin{array}{l}\text { Primer } \\
\text { sequences }\end{array}$ & $\mathbf{H}_{\mathbf{o}}{ }^{a}$ & $\begin{array}{l}\text { Phys. } \\
\text { assign. }\end{array}$ & Reference \\
\hline LEX30 & microsatellite & $\begin{array}{l}\text { GGAGGGTGCAAGGTGCTA } \\
\text { GGCAGGTCAGAAGGGACA }\end{array}$ & 37.5 & & Coogle et al. (1996c) \\
\hline LEX31 & microsatellite & $\begin{array}{l}\text { CCCATTAAGAACTITTCATCCTG } \\
\text { GGCAAGCCCCACAAAATTAT }\end{array}$ & 87.5 & & Coogle et al. (1996c) \\
\hline LEX32 & microsatellite & $\begin{array}{l}\text { CGTAGTAGGGTITTGGGTCC } \\
\text { TTGCGTTTCAATTITAATGAC }\end{array}$ & 50 & & Coogle et al. (1996c) \\
\hline LEX33 & microsatellite & $\begin{array}{l}\text { TITAATCAAAGGATTCAGTTG } \\
\text { TTTCTCTTCAGGTGTCCTC }\end{array}$ & 75 & & Coogle et al. (1996c) \\
\hline LEX34 & microsatellite & $\begin{array}{l}\text { GCGGAGGTAAGAAGTGGTAG } \\
\text { GGCCTAAGATGAGGGTGAA }\end{array}$ & 50 & & Coogle et al. (1997) \\
\hline LEX35 & microsatellite & $\begin{array}{l}\text { CCCAGCATATCAAAGATGTT } \\
\text { GCTCAGTGTACTTCAAGCAG }\end{array}$ & 75 & & Coogle et al. (1997) \\
\hline LEX37 & microsatellite & $\begin{array}{l}\text { GGATTCCTCAACCTCCTAAA } \\
\text { AGGGATAAGTGACCACCAC }\end{array}$ & 25 & & Coogle et al. (1997) \\
\hline LEX38 & microsatellite & $\begin{array}{l}\text { CTGCATTCCCATCATCACAT } \\
\text { TGCCTTGCCTCTTTCTGTTTA }\end{array}$ & 37.5 & & Coogle et al. (1997) \\
\hline LEX39 & microsatellite & $\begin{array}{l}\text { CCTCTGTCCCCACTACTCTC } \\
\text { TTGATCTCCACTCCCAATG }\end{array}$ & 37.5 & & Coogle et al. (1997) \\
\hline LEX40 & microsatellite & $\begin{array}{l}\text { TITGGCCGTTAGTCGTGT } \\
\text { GACAAATCGGAAAGTTGGAA }\end{array}$ & 37.5 & & Coogle et al. (1997) \\
\hline M PZ002 & microsatellite & $\begin{array}{l}\text { GATCCCCCCTATTTTATATACAG } \\
\text { AGGTTCTCATTCTACCTACAAGG }\end{array}$ & 25 & & Breen et al. (1994) \\
\hline SGCV1 & microsatellite & $\begin{array}{l}\text { AGTCACCACCACTCACCTTGT } \\
\text { CCAACACAGGATACGGATGA }\end{array}$ & 37.5 & $?^{d}$ & Godard et al. (1997) \\
\hline SGCV3 & microsatellite & $\begin{array}{l}\text { CCTTGTGGTGAGTTITCCTCTT } \\
\text { CTGCAAAGCTCTGAAGGTC }\end{array}$ & 37.5 & $?^{\mathrm{d}}$ & Godard et al. (1997) \\
\hline SGCV4 & microsatellite & $\begin{array}{l}\text { CGACGCCTCCTCCTAAAC } \\
\text { CAGCTGTGTGCCTITGATTAT }\end{array}$ & 37.5 & $23 q 19$ & Godard et al. (1997) \\
\hline SGCV6 & microsatellite & $\begin{array}{l}\text { GGGCCTGGTTITCCTTCTAA } \\
\text { GCATTTGTGGCCTGTGTCATA }\end{array}$ & 62.5 & $15 q 24$ & Godard et al. (1997) \\
\hline SGCV7 & microsatellite & $\begin{array}{l}\text { GAATTTGAATGTATCTATTCTGAATG } \\
\text { GTGAGTIITCAAGCTGGCATATTC }\end{array}$ & 50 & $18 q 21$ & Godard et al. (1997) \\
\hline SGCV8 & microsatellite & $\begin{array}{l}\text { GAGTTCATTCTTITCGTGGCTG } \\
\text { GGAAACACCCTAAGTGTCCCTTG }\end{array}$ & 37.5 & 19 & Godard et al. (1997) \\
\hline SGCV10 & microsatellite & $\begin{array}{l}\text { CATCCATCCTTTCCAGCTCGATATTC } \\
\text { CAAGACCGTAACTCAGGAGCCC }\end{array}$ & 62.5 & $12 \mathrm{p} 13$ & Godard et al. (1997) \\
\hline SGCV13 & microsatellite & $\begin{array}{l}\text { GGACTAAAGCCCAACCATCCAGC } \\
\text { CTCACCAGTAAGGGGTATGGGGC }\end{array}$ & 75 & $11 q 12$ & Godard et al. (1997) \\
\hline SGCV14 & microsatellite & $\begin{array}{l}\text { CCCCAGTGGTTCCATTTAGATGT } \\
\text { GGGGAGAGCATTITGGTGA }\end{array}$ & 75 & $21 q 13$ & Godard et al. (1997) \\
\hline SGCV16 & microsatellite & $\begin{array}{l}\text { AATTCTCAAATGGTTCAGTGA } \\
\text { СТCССTCССTTCCTTCTA }\end{array}$ & 37.5 & $21 q 13$ & Godard et al. (1997) \\
\hline SGCV17 & microsatellite & $\begin{array}{l}\text { GGCCCAACGTCTATAGAAAGATGT } \\
\text { CCCCCAAATGGCTATTITCTAA }\end{array}$ & 37.5 & $?^{d}$ & Godard et al. (1997) \\
\hline SGCV18 & microsatellite & $\begin{array}{l}\text { TGGGGAAGAGGGATTCAT } \\
\text { AAATGCCAAGCCTATCTATGC }\end{array}$ & 62.5 & 3p13-14 & Godard et al. (1997) \\
\hline SGCV19 & microsatellite & $\begin{array}{l}\text { GCCCCCACCTGCTCCACC } \\
\text { GGGGCAAAGTGGAAATCC }\end{array}$ & 62.5 & $22 q 19$ & Godard et al. (1997) \\
\hline SGCV23 & microsatellite & $\begin{array}{l}\text { GGCTTAAGATATGGGTGAGTAAGG } \\
\text { GCCCACCCTCTTACTIITCTCAA }\end{array}$ & 87.5 & $4 q 27$ & Godard et al. (1997) \\
\hline SGCV24 & microsatellite & $\begin{array}{l}\text { CTACCATTGAAGAGGGGTGGC } \\
\text { GAAACGAGCAGGAAGTGAATCTCC }\end{array}$ & 50 & $11 p 12$ & Godard et al. (1997) \\
\hline SGCV25 & microsatellite & $\begin{array}{l}\text { GCCCATATTAGTAGGACTGTG } \\
\text { GGCCATATTCAGCAGAGCT }\end{array}$ & 37.5 & $1 q 14$ & Godard et al. (1997) \\
\hline SGCV28 & microsatellite & $\begin{array}{l}\text { CTGTGGCAGCTGTCATCTTGG } \\
\text { CCCAATTCCAGCCCAGCTTGC }\end{array}$ & 25 & & Godard et al. (1997) \\
\hline SGCV30 & microsatellite & $\begin{array}{l}\text { ACTGGAGGGGTGAAACAGATTCAGA } \\
\text { GGAAGGGAGGTCATCAGAA }\end{array}$ & 75 & 10qter & $\begin{array}{l}\text { Godard et al. (1997, } \\
\text { 1998) }\end{array}$ \\
\hline
\end{tabular}


LINDGREN ET AL.

Table 1. (Continued)

\begin{tabular}{|c|c|c|c|c|c|}
\hline Locus & Description & $\begin{array}{c}\text { Primer } \\
\text { sequences }\end{array}$ & $\mathrm{H}_{\mathbf{0}}{ }^{\mathrm{a}}$ & $\begin{array}{l}\text { Phys. } \\
\text { assign. }\end{array}$ & Reference \\
\hline SGCV32 & microsatellite & $\begin{array}{l}\text { TGTTCCAAAATGGAGGGTGAGCC } \\
\text { CCACAGGCTCTTAAAACCAGAAGC }\end{array}$ & 62.5 & $?^{d}$ & Godard et al. (1997) \\
\hline VHL20 & microsatellite & $\begin{array}{l}\text { CAAGTCCTCTTACTTGAAGACTAG } \\
\text { AACTCAGGGAGAATCTTCCTCAG }\end{array}$ & 87.5 & & $\begin{array}{l}\text { van Haeringen et al. } \\
\text { (1994) }\end{array}$ \\
\hline VIAS-H34 & microsatellite & $\begin{array}{l}\text { GTATCAGCTTAACAGCTITCTITAAATG } \\
\text { CTCCCGTCTCCTCTCTTGTTC }\end{array}$ & 37.5 & & $\begin{array}{l}\text { Ewen and Matthews } \\
\text { (1994a) }\end{array}$ \\
\hline VIAS-H39 & microsatellite & $\begin{array}{l}\text { AATGTGATTATAGCAGATAGGGTT } \\
\text { CTATCCAATCTTCACAATCATGTA }\end{array}$ & 37.5 & & $\begin{array}{l}\text { Ewen and Matthews } \\
\text { (1994b) }\end{array}$ \\
\hline
\end{tabular}

${ }^{a}$ Observed heterozygosity among the eight sires in the reference pedigree.

${ }^{\text {b}}$ The assignment provided here is based on new experiments, which indicates that these clones are chimeric ( $M$. Breen, unpubl.). Within the chimeric clones, the largest pieces of DNA map to the locations indicated in Breen et al. (1997). However, the sequence surrounding the microsatellite loci have now been confirmed to map to the locations given here.

${ }^{\mathrm{C} C h r o m o s o m e ~ b a n d ~ i d e n t i f i c a t i o n ~ w a s ~ d o n e ~ u s i n g ~ a n ~ o l d ~ i d i o g r a m ~ n o m e n c l a t u r e . ~}$

dPhysical assignment does not fit with linkage data.

of the previous absence of map information for most horse chromosomes. The sum of the length of all linkage groups was $679 \mathrm{cM}$, with an average distance between adjacent markers of $12.6 \mathrm{cM}$. Clearly, a much greater total genetic length is revealed if one takes into account the flanking distances covered by end markers in linkage groups, and the distances covered by the 40 unlinked markers. Using the mean number of informative meioses per marker (89), we can estimate that, on average, our data set allow linkage between two random markers spaced up to $15 \mathrm{cM}$ apart to be detected with an lod score criterion of 3 . Thereby assuming that each of the $2 \times 25=50$ end markers on average cover 15 flanking CMs, the total map length would be about 1425 CM. Furthermore, with the addition of unlinked markers, it is reasonable that the marker set covers well above $1500 \mathrm{cM}$. These can only be seen as very rough estimates as, for instance, some end markers will be close to telomeres al ready.

The linkage groups on ECA3, ECA4, and ECA15 contained five in situ mapped markers, the one on ECA2 had four, whereas ECA1, ECA9, ECA10, ECA11, ECA19, and ECA22 had either two or three physically anchored markers. In most of these cases this allowed determining the orientation of the linkage group along the respective chromosomes. Data from chromosomes with more than one physical tag also allowed a rough analysis of the relationship between genetic and physical distances in the equine genome. Using the approach described in Ellegren et al. (1994), we analyzed this by measuring the physical distance between the most distant anchored markers within linkage groups, expressed as

Table 2. Data for New RFLP Markers

\begin{tabular}{|c|c|c|c|c|c|}
\hline Locus & Enzyme & Alleles & $\begin{array}{l}\text { Polymorphic } \\
\text { fragments (kb) }\end{array}$ & $\begin{array}{c}\text { Observed } \\
\text { heterozygosity }\end{array}$ & Reference \\
\hline FUCA1 & Taql & $\begin{array}{l}A \\
B\end{array}$ & $\begin{array}{l}1.3 \\
1.4\end{array}$ & 0.38 & Fukushima et al. (1985) \\
\hline GLUT1 & Taql & $\begin{array}{l}A \\
B\end{array}$ & $\begin{array}{l}1.6 \\
1.9\end{array}$ & 0.13 & Mueckler et al. (1985) \\
\hline LPL & Taql & $\begin{array}{l}A \\
B\end{array}$ & $\begin{array}{c}3.5+5.4 \\
8.5\end{array}$ & 0.13 & $\begin{array}{l}\text { Harbitz et al. (1992) } \\
\text { Gu et al. (1992) }\end{array}$ \\
\hline MYL1, 3 & Mspl & $\begin{array}{l}A \\
B\end{array}$ & $\begin{array}{l}2.8 \\
3.3\end{array}$ & 0.75 & Seiden et al. (1987) \\
\hline TYR & Taql & $\begin{array}{l}A \\
B\end{array}$ & $\begin{array}{l}2.6 \\
3.4\end{array}$ & 0.25 & Barton et al. (1988) \\
\hline
\end{tabular}


the relative proportion of the genome covered by these markers (measured with a ruler on the karyotype, from the midpoints of in situ assignments), and compared this with the recombinational distance between these markers. A minimum length of linkage groups to beconsidered was set to $20 \mathrm{cM}$. Six groups fulfilled these criteria and gave estimates of $0.70 \mathrm{cM} / \mathrm{Mb}$ (ASB12-ASB8 on ECA1), 0.50 (ASB17ASB13 on ECA2), 0.54 (SGCV18-ASB23 on ECA3), 0.84 (ASB3-SGCV23 on ECA4), 0.73 (ASB6-ASB9 on ECA10), 0.60 (ASB15-HTG6 on ECA15), and 0.83 (I-12-ASB11 on ECA19), the mean being $0.68 \mathrm{cM} /$ $\mathrm{Mb} \pm 0.05$ S.E.

\section{DISCUSSIO N}

This study constitutes the most comprehensive mapping effort so far for the horse genome to date, and is the first to present a preliminary male autosomal linkage map for this species. The number of genetically mapped markers, 100 , far exceeds the sum of that included in earlier overviews (e.g., Sandberg and Andersson 1992) and recent linkage studies (Marklund et al. 1994; Breen et al. 1997; Godard et al. 1997). The majority of the markers genetically mapped in the present study thus represent new linkage assignments. Moreover, for most of the 18 autosomes tagged by linkage groups (i.e., ECA1, ECA2, ECA5, ECA6, ECA7, ECA9, ECA11, ECA13, ECA15, ECA16, ECA18, ECA19, ECA21, and ECA22), the present data either represent the first linkage groups assigned to these chromosomes, or the first to involve more than a single pair of markers.

The total male map distance residing within linkage groups was $679 \mathrm{cM}$, and we estimate that the map covers well above $1500 \mathrm{cM}$ when distances covered by end markers and by unlinked markers are taken into account. It is hard to deduce how large a fraction of the genome is thereby covered, notably because we do not know the total recombinational distance (genetic length) of the equine genome. Total, sex-average distances for other mammalian species range from $\sim 1600 \mathrm{cM}$ (mouse; Davisson and Roderick 1989) to $3500 \mathrm{cM}$ or even higher (human; Weissen bach et al. 1992). In at least some species the male recombination rate is considerably less than that in females (Morton 1991; Ellegren et al. 1994), but this might not be a ubiquitous phenomenon among mammals as suggested by data for cattle and sheep (Crawford et al. 1995; Barendse et al. 1997). The only clue to the genetic length of the equine genome comes from the analysis of meiotic chromosomes (Scott and Long 1980). The number of per cell chiasma in late diplotene or diakinesis among stallions was counted to $54.4 \pm 1.8$, which is comparable to that found in sheep (Chapman and Bruere 1977; Long 1978), but is higher than that in pig (Burt and Bell 1987), cattle, and goat (Logue 1977). The observed number of chiasma in horse would suggest a total male genetic distance of 2720 CM (whereas it would be 2000-2500 cM in pig, cattle, and goat). It is, however, difficult to properly assess genetic lengths from chiasma counts, and also differences between species, as such estimates depend on the precise meiotic stage at which cells are analyzed, something which may vary between studies. As a minimum estimate, it seems reasonable that our map covers at least $50 \%$ of the equine genome.

In a sense, we found it somewhat surprising that the proportion of genotyped markers showing linkage was not higher (140 markers genotyped, 100 linked). Earlier studies of other livestock species have generally noted a higher proportion of linked markers at the corresponding stage of map development (e.g., Ellegren et al. 1994). Although the discrepancy may potentially relate to a difference in the total genetic length of genomes, it seems evident that this mainly reflects an inherent problem in equine genome mapping. The possibility of detecting linkage between markers residing on the same chromosomes will depend obviously on the number of informative meioses shared between the markers. This, in turn, will be a function of marker heterozygosity in the segregating generation and the number of offspring in which transmission can be followed. Because horse gene mapping for reproductive, biological, and practical reasons generally will have to rely on half-sib families (embryo transfer is not yet used in horse), the number of potentially informative meioses will only be half that obtained in analyses of the same number of offspring from full-sib families. This difference is further accentuated if dams are excluded from the family material being genotyped, as was the case in this study, given that it prohibits tracing paternal allele transmission in offspring with the samegenotype as their fathers. Furthermore, as marker heterozygosity in the segregating $F_{1}$ generation in mapping pedigrees of other species is often maximized through crosses between genetical ly divergent parental lines (Beattie 1994), the fact that the economy and practice of horse breeding generally do not allow crosses between divergent breeds implies that a lower proportion of markers will be in heterozygote state in the segregating generation. It could be noted finally that for a majority of the markers used in this study 


\section{LINDGREN ET AL.}
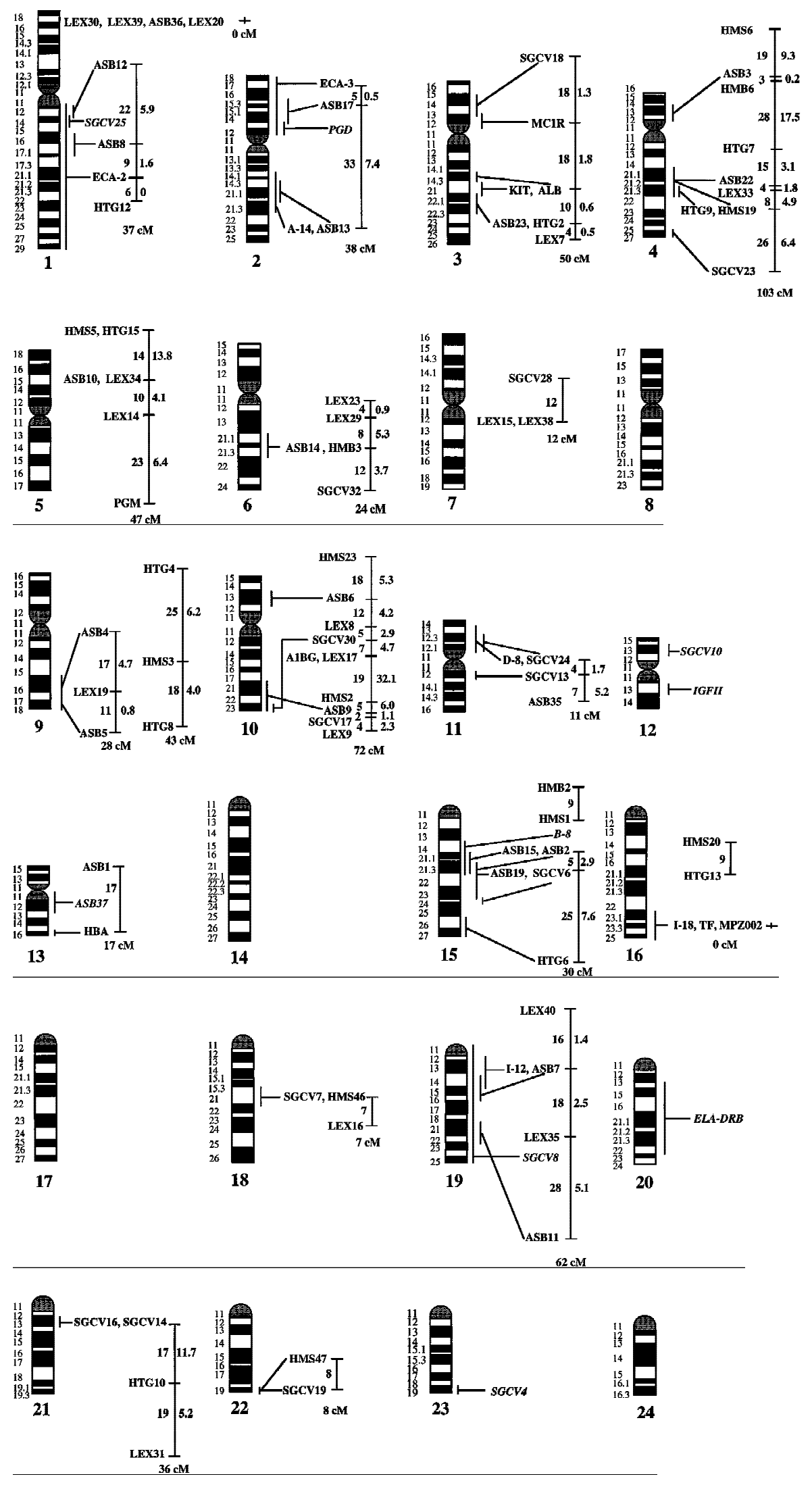

Figure 1 (Continued on facing page.) 

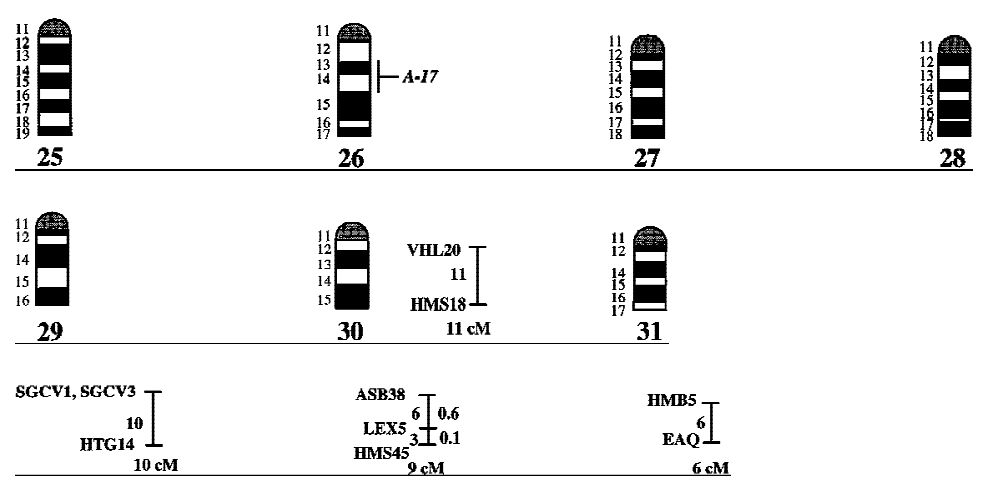

Figure 1 A preliminary male autosomal linkage map of the equine genome. The map depicts all established linkage groups anchored to chromosomes, as well as unassigned linkage groups (bottom). Chromosomal assignments of linkage groups are based on one or more of the markers being physically mapped by in situ hybridization (vertical bars close to chromosomes). The only exceptions are the LEX30-LEX39-MPZ027-LEX20 linkage group on ECA1, the HMS5-HTG15-ASB10-LEX34-LEX14-PGM linkage group on ECA5, the SGCV28-LEX15-LEX38 linkage group on ECA7, the HTG4-HMS3-HTG8 linkage group on ECA9, and HMS20-HTG13 on ECA16, which chromosomal assignments are based on synteny data (Shiue et al. 1998; see also Bailey et al. 1997; Godard et al. 1997). The assignment of the HMB2-HMS1 group to ECA15 is based on a previously observed linkage between one of these markers and physically anchored markers on this chromosome (Godard et al. 1997). The assignment of the group on ECA30 is based on a defined aneuploidy (Bowling et al. 1997b). Values to the left of main vertical bars represent genetic distances between markers expressed as multipoint Kosambi $\mathrm{CM}$, and values to the right are the log 10 odds against reversed order of adjacent markers. Loci shown at the same vertical position had $0 \%$ recombination. Below each linkage group is indicated its total multipoint length. In situ mapped markers genotyped in the family material but not showing linkage are depicted in italics on the map according to their band assignments; their exclusion from linkage groups is evident from the absence of a horizontal line in the vertical bar connecting linked markers. Three markers (ASB13 on ECA2, MC1R on ECA3, and ASB11 on ECA19) known from in situ hybridization to reside within established linkage groups were included in multipoint analyses although only showing a maximum two-point lod score of between 2 and 3.

there is no information available on if the degree of genetic variability differs between breeds, so it cannot be judged if linkage would generally be more easily detected within some breeds than within others.

Some of the linkage assignments made in this study were not in agreement with previously reported map data. The assignments of SGCV17 and SGCV32 to linkage groups on ECA10 and ECA6, respectively (supported by lod scores of 9.98 for SGCV17 and of 7.72 for SGCV32), contradict both their reported FISH mapping to ECA9 (Godard et al. 1997). By somatic cell hybrid mapping, Shiue et al. (1998) similarly placed SGCV17 on ECA10, giving support to our linkage data. New FISH experiments with the SGCV17 and SGCV32 cosmids indicate that they may indeed map to ECA10 and ECA6, respectively (G. Guérin, pers. comm.). Another discrepancy was our observation of close linkage between SGCV1 and SGCV3 (0\% recombination, lod score 11.74). These two markers have been FISH mapped to ECA13 and ECA19, respectively (Godard et al. 1997). Peculiarly, SGCV1 and SGCV3 gave identical microsatellite amplification profiles among unrelated individuals as well as in families (homo/heterozygosity, relative positions of alleles, segregation, etc.), and it appears that they may in fact represent the same locus, their previous disparate in situ assignment being because of human error (G. Guérin, pers. comm.). Yet another discrepancy was the precise location of the marker SGCV30. Physical mapping places it terminal on ECA10q (Godard et al. 1997), however, our linkage data suggests that it is closer to the centromere. All these physical assignments of SGCV markers were done with cosmids and it is possible that the cosmid library contained a significant proportion of chimeric clones, as observed for other equine cosmid libraries (M. Breen, pers. comm.). A few cases of linkage between two markers associated with lod score values slightly above three were likely to represent chance events rather than true linkage. LEX 14 showed $25 \%$ recombination to SGCV30 on ECA10 at lod score 3.01, however, synteny mapping places LEX 14 on ECA5. Similarly, linkages between HMS20 and SGCV18 on ECA3 (24\% recombination, lod score 3.13), and between HTG5 and HMS23 on ECA10 ( $11 \%$ recombination, lod score 3.69 ) may be chance 
events as HMS20 maps to ECA16 and HTG5 to ECA20 in hybrid panel analysis (Shiue et al. 1998). These three linkages have therefore been omitted from the compilation in Figure 1.

Heterologous chromosome painting (ZOO$\mathrm{FISH}$ ) has delineated the overall homologies between the human and equine genomes on the chromosomal level (Raudsepp et al. 1996). In general, chromosomes tend to be well conserved with, for instance, the majority of equine chromosomes corresponding to single human chromosomes (though not necessarily the reverse-a consequence of the horse karyotype containing more chromosomes than the human). Whereas this gross-level information provides an important starting point in the transfer of map information between species, more detailed information on, for example, the positions of genes and gene orders on chromosomes is required for fine-tuned analysis. Two new gene assignments were made in this study, phosphoglucomutase (PGM) to ECA5 and transferrin (TF) to ECA16. Human PGM maps to HSAlp31. According to ZOOFISH (Raudsepp et al. 1996), HSAl corresponds to three different horse chromosomes, ECA2p, ECA5, and ECA30, but it is not known which parts of the human chromosome are homologous to each of the three horse chromosomes. Our mapping of PGM now shows that ECA5 is homologous to at least parts of the $p$ arm of HSAl. As the linkage group on ECA5 is not oriented, we cannot deduce how the HSA1 conservation is arranged along ECA5. The assignment of the TF locus to ECA16 is in agreement with ZOO-FISH data as human TF maps to HSA3q21 and HSA3 corresponds to ECA16 and ECA19. The HSA3q21 band is relatively close to the centromere and given the rather distal location of TF on ECA16, it is possible that the entire $q$ arm of HSA3 corresponds to ECA16, but is orientated reversely. If so, the HSA3-ECA19 homology would involve the $p$ arm of HSA3. Of course, internal rearrangements may occur, but ZOO-FISH data suggest that such events have been rare following the split of the human and equine lineages (Raudsepp et al. 1996).

Only a few mutations causing disease or affecting other important traits have been identified yet in the horse, that is, the adult skeletal muscle sodium channel $\alpha$ subunit gene (SCN4A) associated with hyperkalemic periodic paralysis (HYPP; RudoIph et al. 1992), the catalytic subunit of the DNAdependent protein kinase (DNA-PKCS) associated with severe combined immune deficiency (SCID; Shin et al . 1997), theendothelin receptor B (EDNRB) associated with overo lethal white foal syndrome (OLWS; Santschi et al. 1998), and the melanocyte- stimulating hormone receptor (MC1R) associated with the extension (E) chestnut coat color (Marklund et al. 1996b). All of these mutation identifications have been based on a comparative candidate gene approach, using information from other species in which a similar phenotype and a causative gene has been identified al ready. With the linkage map presented here, it will now become feasible to make genome scans for traits of unknown genetic background in the horse.

In what way should future equine genome mapping go? A first step will obviously have to be extending the present linkage map to reach a nearly complete genome coverage, and to construct a framework map based on highly informative markers ordered with confidence. It is likely that this goal could be reached in the near future by merging maps presently under development in different laboratories, for example, through the international Equine Gene Mapping Workshop collaboration, and by the selected analysis of markers known from synteny mapping to reside on chromosomes with poor coverage (Shiue et al. 1998). In the latter perspective, isolation of markers from chromosomespecific libraries will also be an important tool. During the process of map expansion, there is as well a need for extensive integration of physically anchored markers. The availability of equine BAC Iibraries (e.g., Godard et al . 1998) will allow the identification of large-insert clones containing genetically mapped markers, and the subsequent physical mapping of such clones with FISH. Furthermore, a large number of coding markers should be integrated into the linkage map to facilitate comparative approaches in the search for candidate genes. Recent progress with expressed sequence tags (ESTs) mapping in domestic animals (e.g., Fridolfsson et al . 1997) suggests that this can greatly increase the possibility of exploiting human gene map information across species (e.g., Hasler-Rapacz et al. 1998). As a second step, the development of equine radiation hybrid $(\mathrm{RH})$ panels would greatly facilitate more fine-tuned mapping.

\section{METHODS}

\section{Reference Families}

A Swedish reference pedigree for equine gene mapping has been established in the form of eight half-sib families comprising sires and offspring. Four families are composed of Standardbred (S) trotters and four of Icelandic (I) horses. The number of offspring in the respective families is 25 (S), 27 (S), 31 (S), 31 (I), 31 (I), 31 (I), 40 (S), and 47 (I), totaling 263. Because the number of mares is the same as the number of 
offspring and given that the informativeness of these mares in linkage analysis would be limited, mares were not included in the reference material. As a consequence, only the recombination fractions obtained through male meiosis are followed in this material. Furthermore, this means that $\mathrm{X}$ chromosome linkages were not covered by our data.

\section{Genetic Markers and Genotyping}

A list of genetic markers genotyped in this study is given in Table 1 and includes 121 microsatellites, eight protein polymorphisms, five RFLPs, three blood group polymorphisms, two PCR-RFLPs, and one single-strand conformational polymorphism (SSCP), in total 140 markers. The vast majority of microsatellites was of the (CA $)_{n}$ dinucleotide repeat type, 45 of which had been assigned physically by in situ hybridization (Table 1).

Markers were genotyped essentially as reported in the original references provided in Table 1. Briefly, PCR amplification of microsatellite loci was performed in a MJ Research (PTC-100) thermal cycler in 10- $\mu$ l reactions containing 0.25 units of AmpliTaq DNA polymerase (Perkin-Elmer), $200 \mu \mathrm{m}$ dNTPs, $1.5 \mathrm{~mm} \mathrm{MgCl} 2,50 \mathrm{~mm} \mathrm{KCl}, 10 \mathrm{~mm}$ Tris- $\mathrm{HCl}(\mathrm{pH} \mathrm{8.3)}$, $0.001 \%$ (wt/vol) gelatin, $100 \mathrm{ng}$ of genomic DNA, and $1-3$ pmoles of each primer. $5^{\prime}$ end labeling of one primer in each primer pair was carried out in $25-\mu \mathrm{l}$ reactions using $0.1 \mu \mathrm{Ci}$ of $\left[\gamma^{32} \mathrm{P}\right]$ per pmole primer, 10 units of T4 polynucleotide kinase (New England Biolabs, Beverly, MA), 70 mm Tris- $\mathrm{HCl}$ (pH 7.6), $10 \mathrm{~mm} \mathrm{M} \mathrm{MCl}_{2}$, and $5 \mathrm{~mm}$ DTT. The standard PCR profile consisted of one cycle of $94^{\circ} \mathrm{C}$ for $3 \mathrm{~min}, 58^{\circ} \mathrm{C}$ for $30 \mathrm{sec}$, and $72^{\circ} \mathrm{C}$ for $1 \mathrm{~min}$, followed by $29 \mathrm{cycles}$ of $94^{\circ} \mathrm{C}$ for $30 \mathrm{sec}, 55^{\circ} \mathrm{C}$ for 30 $\mathrm{sec}$, and $72^{\circ} \mathrm{C}$ for $30 \mathrm{sec}$. After the final cycle a prolonged extension step of $10 \mathrm{~min}$ was included. The PCR products were mixed with loading buffer (95\% formamide, $0.05 \%$ xylene cyanol FF, $0.05 \%$ bromophenol blue, 0.02 м EDTA) and electrophoresed for 1-2 $\mathrm{hr}$ in $6 \%$ denaturating polyacrylamide gels (Sequagel XR, National Diagnostics, Atlanta, GA). Subsequently, gels were soaked in $10 \%$ acetic acid, dried at $70^{\circ} \mathrm{C}$, and exposed to autoradiographic films overnight.

Five heterologous mammalian cDNA probes, fucosidase 1 (FUCA1), glucose transporter 1 (GLUT1), lipoprotein lipase (LPL), myosin light chain 1 and 3 (MYL1,3), and tyrosinase (TYR), were used for RFLP analysis (Table 2). Fifteen micrograms of genomic DNA was digested with either Mspl, Pvull, or Taql (Promega, Madison, WI), separated in 0.9\% agarose gels, and transferred to Hybond $\mathrm{N}+$ membranes (Amersham Pharmacia Biotech, Uppsala, Sweden). Hybridization was made in $0.26 \mathrm{M} \mathrm{Na}_{2} \mathrm{HPO}_{4}, 7 \% \mathrm{SDS}, 5 \%$ dextran sulfate, $1 \%$ bovine serum albumin, and $0.2 \mathrm{mg} / \mathrm{ml}$ salmon sperm DNA at $65^{\circ} \mathrm{C}$, using probes labeled with $\left[\alpha^{32} \mathrm{P}\right] \mathrm{dCTP}$ by nick translation. Membranes were washed at a final stringency of $0.2 \times$ SSC at $60^{\circ} \mathrm{C}$ and exposed to autoradiographic films for 2-6 days.

\section{Linkage A nalysis}

Linkage between markers was analyzed using the program CRIMAP, version 2.4 (Green et al. 1990). First, the TWOPOINT option of CRIMAP was used to detect pair-wise linkages. An lod score threshold of three was set as criterion for significant linkage. For multipoint analysis of larger linkage groups we used the option BUILD to select markers to be used as a framework for the continuing ordering of additional markers; the order of selected markers was supported by an lod score of three or higher. The option ALL was used subsequently to incorporate the rest of the markers, one at a time. The FLIPS and FIXED options were used finally for evaluating the statistical support of the proposed order and the distance between markers, respectively. All multipoint distances are expressed as Kosambi CM. For smaller linkage groups $(\leqslant 5$ markers) we started with the ALL option and then ran the FLIPS2 option. Genotype data were checked for typing errors using the CHROMPIC option, as a means to identify unlikely double recombinants.

\section{A CKN O WLEDG MENTS}

The technical assistance of Sigridur Björnsdottir, Lena Oscarsson, and Eva Pettersson is acknowledged. Leif Andersson is acknowledged for support during the project. Financial support was obtained from the Swedish Research Council for Agriculture and Forestry, the Erik Philip-Sörensens Foundation, the Trygger foundation, and The Royal Swedish Academy of Agriculture and Forestry.

The publication costs of this article were defrayed in part by payment of page charges. This article must therefore be hereby marked "advertisement" in accordance with 18 USC section 1734 solely to indicate this fact.

\section{REFEREN CES}

Andersson, L. and K. Sandberg. 1982. A linkage group composed of three coat colour genes and three serum protein loci in horses. J. Hered. 73: 91-94.

Andersson, L., C.S. Haley, H. Ellegren, S.A. Knott, M. Johansson, K. Andersson, L. Andersson-Eklund, I. Edfors-Lilja, M. Fredholm, I. Hansson, J. Håkansson, and K. Lundström. 1994. Genetic mapping of quantitative trait loci for growth and fatness in pigs. Science 263: 1771-1774.

Ansari, H.A., R. Hediger, R. Fries, and G. Stranzinger. 1988. Chromosomal localization of the major histocompatibility complex of the horse (ELA) by in situ hybridization. Immunogenetics 28: 362-364.

Bailey, E., R.C. Reid, L.C. Skow, K. Mathiason, T.L. Lear, and T.C. McGuire. 1997. Linkage of the gene for equine combined immunodeficiency disease to microsatellite markers HTG8 and HTG4; synteny and FISH mapping to ECA9. Anim. Genet. 28: 268-273.

Bailey, E., C. Stormont, Y. Suzuki, and A. Trommershausen Smith. 1979. Linkage of loci controlling alloantigens on red blood cells and lymphocytes in the horse. Science

204: 1317-1319.

Barendse, W., D. Vaiman, S.J. Kemp, Y. Sugimoto, S.M. Armitage, J.L. Williams, H.S. Sun, A. Eggen, M. Agaba, S.A. Aleysin et al. 1997. A medium-density genetic linkage map of the bovine genome. Mamm. Genome 8: 21-28.

Barton, D.E., B.S. Kwon, and U. Francke. 1988. Human tyrosinase gene, mapped to chromosome 11 (q14-q21), defines second region of homology with mouse chromosome 7. Genomics 3: 17-24. 
LINDGREN ET AL.

Beattie, C.W. 1994. Livestock genome maps. Trends Genet. 10: 334-338.

Binns, M.M., N.G. Holmes, A. Holliman, and A.M. Scott. 1995. The identification of polymorphic microsatellite loci in the horse and their use in thoroughbred parentage testing. Br. Vet. J. 151: 9-15.

Bowling, A.T. and R.S. Clark. 1985. Blood group and protein polymorphism gene frequencies for seven breeds of horses in the United States. Anim. Blood Groups Biochem. Genet. 16: $93-108$.

Bowling, A.T., A.M. Scott, J. Flint, and J.B. Clegg. 1988. Novel al pha haemoglobin haplotypes in horses. Anim. Genet. 19: 87-101.

Bowling, A.T., M. Breen, B.P. Chowdhary, K. Hirota, T. Lear, L.V. Millon, F.A.P. Leon, T. Rausepp, and G. Stranzinger. 1997a. International System for Cytogenetics Nomenclature of the domestic Horse. Chromosome Res. 5: 433-443.

Bowling, A.T., L.V. Millon, and S. Dileanis. 1997b. Physical mapping of genetic markers to chromosome 30 using a trisomic horse and evidence for maternal origin of the extra chromosome. Chromosome Res. 5: 429-431.

Breen, M., P. Downs, Z. Irvin, and K. Bell. 1994. Six equine dinucleotide repeats: Microsatellites MPZOO2, 3, 4, 5, 6 and 7. Anim. Genet. 25: 124.

Breen, M., G. Lindgren, M.M. Binns, J. Norman, Z. Irvin, K. Bell, K. Sandberg, and H. Ellegren. 1997. Genetical and physical assignments of equine microsatellites-first integration of anchored markers in horse genome mapping. Mamm. Genome 8: 267-273.

Burt, A. and G. Bell. 1987. Mammalian chiasma frequencies as a test of two theories of recombination. Nature 326: 803-805.

Chapman, H.M. and A. Bruere. 1977. Chromosome morphology during meiosis of normal and Robertsonian translocation-carrying rams (Ovis aries). Can. J. Genet. Cytol. 19: 93-102.

Charlier, C., W. Coppieters, F. Farnir, L. Grobet, P.L. Leroy, C. Michaux, M. M ni, A. Schwers, P. Vanmanshoven, R. Hanset, and M. Georges. 1995. The mh gene causing double-muscling in cattle maps to bovine chromosome 2 . Mamm. Genome 6: 788-792.

Coogle, L., E. Bailey, R. Reid, and M. Russ. 1996a. Equine dinucleotide repeat polymorphisms at loci LEX002, -003, $-004,-005,-007,-008,-009,-010,-011,-013$ and -014. Anim. Genet. 27: 126-127.

Coogle, L., R. Reid, and E. Bailey. 1996b. Equine dinucleotide repeat loci LEX015-024. Anim. Genet. 27: 217-218.

1996c. Equine dinucleotide repeat loci from LEX025 to LEX033. Anim. Genet. 27: 289-290.
-_-. 1997. Equine dinucleotide repeat loci LEX034-LEX048. Anim. Genet. 28: 308-322.

Crawford, A.M., K.G. Dodds, A.J. Ede, C.A. Pierson, G.W. Montgomery, H.G. Garmonsway, A.E. Beattie, K. Davies, J.F. Maddox, S.W. Kappes et al. 1995. An autosomal genetic linkage map of the sheep genome. Genetics 140: 703-724.

Davisson, M.T. and T.H. Roderick. 1989. Linkage map. In Genetic variants and strains of the laboratory mouse, 2 nd ed. (ed. M.F. Lyon and A.G. Searle), pp. 416-427. Gustav Fischer Verlag, Stuttgart, Germany.

Dawson, D.M. and S. Jaeger. 1970. Heterogeneity of phosphoglucomutase. Biochem. Genet. 4: 1-9.

Ellegren, H., M. Johansson, K. Sandberg, and L. Andersson. 1992. Cloning of highly polymorphic microsatellites in the horse. Anim. Genet. 23: 133-142.

Ellegren, H., B.P. Chowdhary, M. Johansson, L. Marklund, M. Fredholm, I. Gustavsson, and L. Andersson. 1994. A primary linkage map of the porcine genome reveals a low rate of genetic recombination. Genetics 137: 1089-1100.

Ewen, K.R. and M.E. Matthews. 1994a. VIAS-H17 and VIAS-H34: Two new polymorphic equine microsatellite loci. Anim. Genet. 25: 63.

1994b. VIAS-H39, an equine tetranucleotide microsatellite repeat polymorphism. Anim. Genet. 25: 433.

Fraser, D.G. and E. Bailey. 1996. Demonstration of three DRB loci in a domestic horse family. Immunogenetics 44: 441-445.

Fridolfsson, A.-K., T. Hori, A.-K. Winterø, M. Fredholm, M. Yerle, A. Robic, L. Andersson, and H. Ellegren. 1997. Expansion of the pig comparative map by expressed sequence tags (EST) mapping. Mamm. Genome 8: 907-912.

Fukushima, H., J.R. de Wet, and S.J. O'Brien. 1985. Molecular cloning of a CDNA for human $\alpha$-L-fucosidase. Proc. Natl. Acad. Sci. 82: 1262-1265.

Gahne, B. 1966. Studies on the inheritance of electrophoretic forms of transferrins, albumins, prealbumins and plasma esterases of horses. Genetics 53: 681-694.

Georges, M., D. Nielsen, M. Mackinnon, A. Mishra, R. Okimoto, A.T. Pasquino, L.S. Sargeant, A. Sorensen, M.R. Steele, X. Zhao, J.E. Womack, and I. Hoeschele. 1995. Mapping quantitative trait loci controlling milk production in dairy cattle by exploiting progeny testing. Genetics 139: 907-920.

Godard, S., D. Vaiman, A. Oustry, M. Nocart, M. Bertaud, S. Guzylack, J.C. Mériaux, E.P. Cribiu, and G. Guérin. 1997. Characterization, genetic and physical mapping analysis of 36 horse plasmid and cosmid-derived microsatellites. Mamm. Genome 8: 745-750.

Godard, S., L. Schibler, A. Oustry, E.P. Cribiu, and G. Guérin. 1998. Construction of a horse BAC library and 
cytogenetical assignment of 20 type I and type II markers. Mamm. Genome 9: 633-637.

Green, P., K.A. Falls, and S. Crooks. 1990. Documentation for CRI-MAP, version 2.4. Washington University School of Medicine, St. Louis, MO.

Gu, F., I. Harbitz, B.P. Chowdhary, R. Chaudhary, and I. Gustavsson. 1992. Localization of the 6-phosphogluconate dehydrogenase (PGD) gene in horses by in situ hybridization. Hereditas 117: 93-95.

Gu, F., I. Harbitz, B.P. Chowdhary, W. Davies, and I. Gustavsson. 1992. Mapping of the porcine lipoprotein lipase (LPL) gene to chromosome 14q12-q14 bands by in situ hybridisation. Cytogenet. Cell Genet 59: 63-64.

Guérin, G. and M. Bertaud. 1996. Characterization of two polymorphic horse microsatellites: HMS15 and HMS20. Anim. Genet. 27: 123.

Guérin, G., M. Bertaud, and Y. Amigues. 1994. Characterization of seven new horse microsatellites: HMS1, HMS2, HMS3, HMS5, HMS6, HMS7 and HMS8. Anim. Genet. 25: 62.

Harbitz, I., T. Kristensen, and W. Davies. 1992. Isolation and sequencing of porcine lipoprotein lipase cDNA and its use in multiallelic restriction fragment length polymorphism detection. Anim. Genet. 23: 517-522.

Hasler-Rapacz, J., H. Ellegren, A.-K. Fridolfsson, B. Kirkpatrick, S. Kirk, L. Andersson, and J. Rapacz. 1998. Identification of a mutation in the low density lipoprotein receptor gene associated with recessive familial hypercholesterolemia in swine. Am. J. Med. Genet. 76: 379-386.

Irvin, Z., J. Giffard, R. Brandon, M. Breen, and K. Bell. 1998. Equine dinucleotide repeat polymorphisms at loci ASB21, 23, 25 and 37-43. Anim. Genet. 29: 67.

Juneja, R.K., B. Gahne, and A. Stratil. 1987. Polymorphic plasma postal bumins of some domestic animals (pig PO2, horse $\mathrm{Xk}$, and dog Pa proteins) identified as homologous to human plasma alpha 1B-glycoprotein. Anim. Genet. 18: $119-124$.

Kappes, S.M., J.W. Keele, R.T. Stone, R.A. McGraw, T.S. Sonstegard, T.P. Smith, N.L. Lopez-Corrales, and C.W. Beattie. 1997. A second-generation linkage map of the bovine genome. Genome Res. 7: 235-249.

Lear, T.L., Z. Irwin, R. Brandon, K. Bell, K. Mathiason, and E. Bailey. 1998. Physical assignment of microsatellite markers to horse chromosomes using FISH. In Proceedings, plant and animal genome VI, pp. 162. San Diego, CA.

Lear, T.L., M.H. Adams, K.J. McDowell, N.D. Sullivan, L. Coogle, E. Ferguson Jr., T.M. Chambers, and E. Bailey. 1997. Chromosomal location of the genes for ESR, ETS2, GOT2, $\mathrm{KIT}, \mathrm{MX} 1$ and PGR in the horse, Equus caballus. In Proceedings of the 10th North American colloquium on gene mapping and cytogenetics in human and domestic species, p. 24. Apalachicola, FL.

Lindgren, G., H. Persson, and H. Ellegren. 1998. Five equine dinucleotide microsatellite loci HTG17, HTG20, HTG21, HTG28 and HTG31. Anim. Genet. (in press).

Long, S.E. 1978. Chiasma counts and non-disjunction frequencies in a normal ram and in rams carrying the Massey I $\left(t_{1}\right)$ Robertsonian translocation. J. Reprod. Fertil. 53: 353-356.

Logue, D.N. 1977. Meiosis in the domestic ruminants with particular reference to Robertsonian translocations. Ann. Genet. Sel. Anim. 9: 493-507.

MacLennan, D.H., C. Duff, F. Zorzato, J. Fujii, M. Philips, R.G. Korneluk, W. Frodis, B.A. Britt, and R.G. Worton. 1990. Ryanodine receptor gene is a candidate for predisposition to malignant hyperthermia. Nature 343: 559-561.

Marklund, S., H. Ellegren, S. Eriksson, K. Sandberg, and L. Andersson. 1994. Parentage testing and linkage analysis in the horse using a set of highly polymorphic microsatellites. Anim. Genet. 25: 19-23.

Marklund, L., M. Johansson Moller, B. Høyheim, W. Davies, M. Fredholm, R.K. Juneja, P. Mariani, W. Coppieters, H. Ellegren, and L. Andersson. 1996a. A comprehensive linkage map of the pig based on a wild pig-Large White intercross. Anim. Genet. 27: 255-269.

Marklund, L., M. Johansson Moller, K. Sandberg, and L. Andersson. 1996b. A missense mutation in the gene for melanocyte-stimulating hormone receptor (MC1R) is associated with the chestnut coat color in horses. Mamm. Genome 7: 895-899.

Marti, E., M. Breen, P. Fischer, J. Swinburne, and M.M. Binns. 1998. Six new cosmid derived and physically mapped equine dinucleotide repeat microsatellites. Anim. Genet. 29: 236-238.

Mellersh, C.S., A.A. Langston, G.M. Acland, M.A. Fleming, K. Ray, N.A. Wiegand, L.V. Francisco, M. Gibbs, G.D. Aguirre, and E.A. Ostrander. 1997. A linkage map of the canine genome. Genomics 46: 326-336.

Montgomery, G.W., E.A. Lord, J.M. Penty, K.G. Dodds, T.E. Broad, L. Cambridge, S.L.F. Sunden, R.T. Stone, and A. Crawford. 1994. The Booroola fecundity (FecB) gene maps to sheep chromosome 6. Genomics 22: 148-153.

Morton, N.E. 1991. Parameters of the human genome. Proc. Natl. Acad. Sci. 88: 7474-7476.

Mueckler, M., C. Caruso, S.A. Baldwin, M. Panico, I. Blench, H.R. Morris, W.J. Allard, G.E. Lienhard, and H.F. Lodish. 1985. Sequence and structure of a human glucose transporter. Science 229: 941-945.

Oakenfull, E.A., V.J. Buckle, and J.B. Clegg. 1993. Localization of the horse (Equus caballus) $\alpha$-globin gene 
LINDGREN ET AL.

complex to chromosome 13 by fluorescence in situ hybridization. Cytogenet. Cell Genet. 62: 136-138.

Raudsepp, T., L. Frönicke, H. Scherthan, I. Gustavsson, and B.P. Chowdhary. 1996. Zoo-FISH delineates conserved chromosomal segment in horse and man. Chromosome Res. 4: 218-225.

Raudsepp, T., K. Otte, B. Rozell, and B.P. Chowdhary. 1997. FISH mapping of the IGF2 gene in horse and donkey-detection of homoeology with HSAll. Mamm. Genome 8: 569-572.

Rohrer, G.A., L.J. Alexander, Z. Hu, T.P. Smith, J.W. Keele, and C.W. Beattie. 1996. A comprehensive map of the porcine genome. Genome Res. 6: 371-391.

Rudolph, J.A., S.J. Spier, G. Byrns, C.V. Rojas, D. Bernoco, and E.P. Hoffman. 1992. Periodic paralysis in Quarter horses: A sodium channel mutation disseminated by selective breeding. Nat. Genet. 2: 144-147.

Sakagami, M., T. Tozaki, S. Mashima, K. Hirota, and H. Mukoyama. 1995. Equine parentage testing by microsatellite locus at chromosome 1q2.1. Anim. Genet. 26: $123-124$.

Sandberg, K. 1968. Genetic polymorphism in carbonic anhydrase from horse erythrocytes. Hereditas 60: 411-412.

- - - 1973. The D blood group system of the horse. Anim. Blood Groups Biochem. Genet. 4: 193-205.

Sandberg, K. and L. Andersson. 1992. Horse (Equus caballus). In Genetic maps, 6th ed. (ed. S.J. O'Brien), pp. 4.276-4.278. Cold Spring Harbor Laboratory Press, Cold Spring Harbor, NY.

Santschi, E.M., A.K. Purdy, S.J. Valberg, P.D. Vrotsos, H. Kaese, and J.R. Mickelson. 1998. Endothelin receptor B polymorphism associated with lethal white foal syndrome in horses. Mamm. Genome 9: 306-309.

Scott, I.S. and S.E. Long. 1980. An examination of chromosomes in the stallion (Equus caballus) during meiosis. Cytogenet. Cell Genet. 26: 7-13.

Seidel, U., E. Bober, B. Winter, S. Lenz, P. Lohse, and H.H. Arnold. 1987. The complete nucleotide sequences of CDNA clones coding for human myosin light chains 1 and 3. Nucleic Acids Res. 15: 4989.

Shin, E.K., L.E. Perryman, and K. Meek. 1997. A kinase-negative mutation of DNA-PK ${ }_{C S}$ in equine SCID results in defective coding and signal joint formation. J. Immunol. 158: 3565-3569.

Shiue, Y., L.A. Bickel, A.R. Caetano, L.V. Millon, R.S. Clark, M.L. Eggleston, R. Michelmore, E. Bailey, G. Guérin, S. Godard, J.R. Mickelson, S.J. Valberg, J.D. Murray, and A.T. Bowling. 1998. A synteny map of the horse genome comprised of 240 microsatellite and RAPD markers. Anim. Genet. (in press).
Tozaki, T., M. Sakagami, S. Mashima, K. Hirota, and H. Mukoyama. 1995. ECA-3: Equine (CA) repeat polymorphism at chromosome 2p1.3-4. Anim. Genet. 26: 283.

Vaiman, D., L. Schibler, F. Ourgeois, A. Oustry, Y. Amigues, and E.P. Cribiu. 1996. A genetic linkage map of the male goat genome. Genetics 144: 279-305.

van Haeringen, H., A.T. Bowling, M.L. Stott, J.A. Lenstra, and K.A. Zwaagstra. 1994. A highly polymorphic horse microsatellite locus: VHL20. Anim. Genet. 25: 207.

Vega-Pla, J.L., J.J. Garrido, G. Dorado, and D.F. de Andrés-Cara. 1996. Three new polymorphic equine microsatellites: HLM2, HLM3, HLM5. Anim. Genet. 27: 215.

Weissenbach, J., G. Gyapay, C. Dib, A. Vignal, J. Morisette, P. Millasseau, G. Vaysseix, and M. Lathrop. 1992. A second-generation linkage map of the human genome. Nature 359: 794-801.

Received June 30, 1998; accepted in revised form August 5, 1998. 


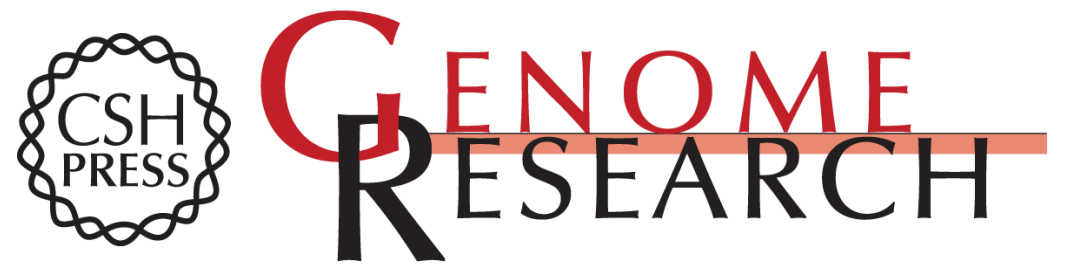

\section{A Primary Male Autosomal Linkage Map of the Horse Genome}

Gabriella Lindgren, Kaj Sandberg, Helena Persson, et al.

Genome Res. 1998 8: 951-966

Access the most recent version at doi:10.1101/gr.8.9.951

References This article cites 70 articles, 14 of which can be accessed free at:

http://genome.cshlp.org/content/8/9/951.full.html\#ref-list-1

License

Email Alerting Receive free email alerts when new articles cite this article - sign up in the box at the Service top right corner of the article or click here.

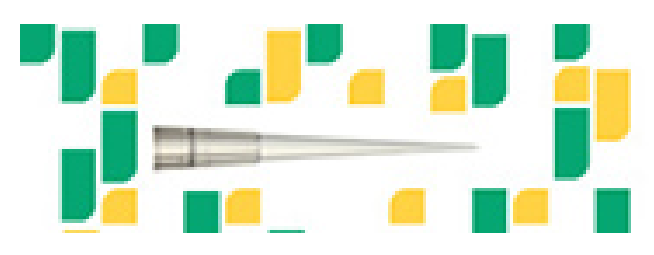

To subscribe to Genome Research go to: https://genome.cshlp.org/subscriptions 